Hautarzt 2010 • 61:97-98

DOI 10.1007/s00105-010-1918-7

Online publiziert: 28. Januar 2010

(c) Springer-Verlag 2010

\author{
H.C. Korting ${ }^{1}$ P. Lehmann ${ }^{2}$ \\ ${ }^{1}$ Klinik und Poliklinik für Dermatologie und Allergologie, \\ Klinikum der Universität München \\ ${ }^{2}$ Kompetenzzentrum Hautkrebs, Zentrum für Dermatologie, \\ Allergologie und Dermatochirurgie, Wuppertal
}

\title{
Acne vulgaris
}

in letzter Zeit dem größten Wandel unterworfen waren.

Dies gilt wohl zuallererst für die Pathogenese. Hier hat es G. Plewig unternommen, sozusagen eine Summe seines wissenschaftlichen Wirkens auf einem seiner Haupttätigkeitsfelder zu geben. Dabei wird es denjenigen, welcher die Entfaltung seines wissenschaftlichen Werkes über die Jahre verfolgt hat, nicht überraschen, dass weiterhin unterschiedliche Phänomene gleichermaßen als bedeutsam erachtet werden, nämlich „die Verhornungsstörung, die erhöhte Talgproduktion, die Besiedelung mit Propionibacterium acnes oder entzündliche Phänomene“. Das Proprium liegt dabei insbesondere auch auf der relativen Gewichtung dieser Aspekte, des Weiteren auf der Herausarbeitung von Einzelaspekten, die der Leser vermutlich, wenn er nicht zu den absoluten Spezialisten gehört, kaum vor Augen gehabt haben dürfte, wie etwa die Interaktion zwischen Talgdrüsen und Bindegewebe, nach aktueller Auffassung verknüpft über den Fibroblasten-Wachstumsfaktor-Rezeptor-2 (FGFR2). Als ein Faktor unter vielen, und zwar als ein solcher, an dem es womöglich anzusetzen gilt, wird von Plewig die Ernährung bezeichnet. Hier und nicht bei anderen Aspekten stellt er eine Frage in den Raum, nachdem auch er darauf hinweist, dass epidemiologische Studien heute hindeuten „auf Auslösung von Akne durch hyperglykämische Nahrung und Milchkonsum“. Seine Frage lautet: „Wo geht die Entwicklung hin?"

Genau diese Frage wird umfassend in dem Aufsatz von B. Melnik mit dem Titel: „Acne vulgaris: Rolle der Diät“ ab- gehandelt. Hier wird bereits in der $\mathrm{Zu}$ sammenfassung eine Kernthese formuliert, die da lautet: „Akne ist das sichtbare metabolische Syndrom der Haut", wobei auch gleich eine Begründung in Form der folgenden Einlassung geliefert wird: „durch übersteigerte Wachstumsfaktorsignale westlicher Fehlernährung“. Vor diesem Hintergrund wird dann auch gleich ein neuer nosologischer Terminus vorgeschlagen, nämlich: „Acne alimentaris“, was nach Auffassung des Autors auch unmittelbare Konsequenzen hat, insofern er „diätetische Intervention“ fordert. Dieser Aufruf bleibt im Übrigen nicht im Ungewissen stehen. Ganz konkret fordert Melnik: „Es sollten alle Anstrengungen unternommen werden, den insulinotropen Effektormechanismus der Milch zu eliminieren, um die Prävalenz der Akne und weiterer ernster konsekutiver Zivilisationskrankheiten mit überhöhter fehlernährungsbedingter onkogener Signaltransduktion zu vermindern.“ Nachdem wir lange von dem Diktum geprägt waren, dass Acne vulgaris im Grunde kaum etwas mit Ernährung zu tun habe, in Sonderheit nicht mit dem immer wieder diskutierten Genuss von (Milch)Schokolade, deutet sich hier ein möglicher Trendwandel an, der umso bedenkenswerter erscheint, als G. Plewig gerade in diesem Punkt signalisiert, dass ein Umdenken womöglich in der Tat nottut. Sollte sich die hier so engagiert von B. Melnik vertretene These langfristig in der medizinischen Wissenschaft etablieren, so könnte das vorliegende Heft der Zeitschrift „Der Hautarzt“ sozusagen im goetheschen Sinne ein Valmy der Dermatologiegeschichte markieren. 
Dennoch bzw. in gewisser Weise umso mehr bedarf es im Kontext der Pathogenese und in Teilen auch der Behandlung der Acne vulgaris einer umfassenden Betrachtung der Rolle der Hormone, eine Aufgabe, der sich C. Zouboulis unterzogen hat. Wie so oft bei Fragen des Hormonhaushalts und ihren medizinisch relevanten Implikationen handelt es sich um eine komplexe Materie, wobei aber aus der Sicht des Autors 2 Fakten besondere Beachtung verdienen:

- „Der Beginn der Akne fällt häufig mit der Adrenarche, und zwar mit Beginn der Synthesesteigerung des Vorstoffes von Testosteron Dehydroepiandrosteronsulfat durch die Nebennierenrinde, zusammen."

- Des Weiteren „zeigen Talgdrüsenzellen aus Akneregionen eine höhere Ansprechbarkeit auf Androgene als Talgdrüsenzellen aus anderen Körperarealen".

Was die Frage der Rolle der Hormone angeht, darf der Blick im Übrigen nicht auf körpereigene Hormone verengt werden. Dies macht eine der Kernthesen der Arbeit deutlich, die lautet: „Lipide und Hormone in der Diät sowie Neuropeptide können zu der Entstehung einer Akne beitragen."

So wichtig ein vertieftes Verständnis der Pathogenese einer Krankheit für das Krankheitsmanagement insgesamt ist, bleibt doch nicht außer Acht zu lassen, dass trotz aller etwaigen Vorbeugungsmaßnahmen die Krankheit in vielen Fällen bereits manifest als Problem an den Arzt und hier speziell den Hautarzt herangetragen wird. Will er sich dieser vielfältigen Herausforderung in zeitgemäßer Weise stellen, so wird er heute keineswegs nur auf medikamentöse Therapie zurückgreifen, die im Übrigen in letzter Zeit eher durch Schritt- als durch Durchbruchinnovationen gekennzeichnet war, wie sie vor längerer Zeit beispielsweise in der Einführung des systemischen Isotretinoin bestanden hat.

Aktuell ist eine rasante Entwicklung bei den physikalischen Therapieverfahren der Akne zu verzeichnen. Dies macht der Aufsatz von C. Borelli und H.C. Korting deutlich, der sich mit der Behandlung mit ungebündeltem und gebündeltem Licht (La- ser) sowie der photodynamischen Therapie und schließlich dem Peeling auseinandersetzt. In dem gesamten Feld der physikalischen Therapieverfahren stellt sich eine ganze Fülle neuer Ansätze dar, sodass man hier möglicherweise von einer „neuen Unübersichtlichkeit“ sprechen könnte. Immerhin kann man schon heute sagen, dass eine Reihe von vielversprechenden neuen Verfahren entwickelt worden ist, auch dann, wenn die endgültige Einordnung der Relevanz zum gegenwärtigen Zeitpunkt noch schwerfällt, nicht zuletzt deshalb, weil nicht selten ein Mangel an wirklich aussagekräftigen Studien zur Wirksamkeit und Verträglichkeit gegeben ist. In jedem Falle schließt sich bei der Lichtanwendung ein Kreis: Sie, die vor Jahrzehnten in anderer Form schon einmal weit verbreitet war, findet heute wieder großes Interesse.

Großes Interesse findet auch immer das Thema Acne vulgaris und Kosmetik. Lag hier lange der Akzent auf der Furcht vor unerwünschten Wirkungen von Kosmetika, hat sich dies in der letzten Zeit ins Gegenteil verkehrt: Wir wissen heute, dass frühere Sorgen betreffend akneigener Kosmetika respektive Kosmetikainhaltsstoffen übertrieben gewesen sind, was uns als Dermatologen freilich nicht von der Aufgabe entbindet, wachsam zu bleiben. Darüber hinaus kann man heute aber auch feststellen, dass Kosmetika tatsächlich zur begleitenden Reinigung und Pflege der Haut bei Acne vulgaris bzw. entsprechender Krankheitsneigung von Bedeutung sind. Wie in dem Aufsatz von H.C. Korting, C. Borelli und C. Schöllmann ausgeführt wird, kann man heute auch im Kontext der Acne vulgaris durchaus von einer beginnenden Entfaltung des Konzeptes der evidenzbasierten Kosmetik sprechen. Der Rückgriff auf eine ganze Reihe von Prinzipien erscheint heute durch Studien abgesichert. Im Kontext der Hautreinigung ist hier auf die Rolle des leicht sauren $\mathrm{pH}$-Werts zu verweisen, im Kontext der Hautpflege auf bestimmte aktive kosmetische Ingredienzien.

Wer die angesprochenen Kapitel rezipiert hat, wird zu der Auffassung gelangen, dass gerade auch die Acne vulgaris eine nosologische Entität repräsentiert, die in den letzten Jahren von umfassender wissenschaftlicher Forschung im Labor wie in der Klinik gleichermaßen profitiert hat - zum Wohle der in einem bestimmten Lebensalter so häufigen Patienten, zudem einem Lebensalter, in dem nicht zuletzt auch ästhetische Probleme erhebliche psychosoziale Folgen haben können.

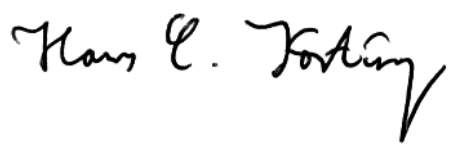

Prof. Dr. H.C. Korting

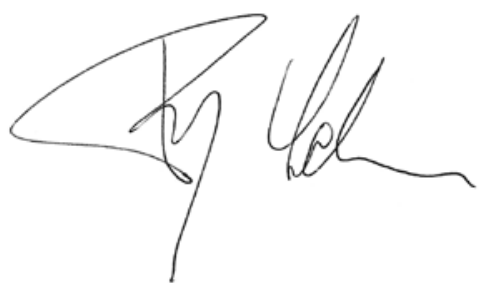

Prof. Dr. P. Lehmann

\section{Korrespondenzadresse}

Prof. Dr. H.C. Korting

Klinik und Poliklinik für Dermatologie und Allergologie, Klinikum der Universität München Frauenlobstr. 9-11, 80337 München

HansChristian.Korting@med.uni-muenchen.de

Interessenkonflikt. Der korrespondierende Autor weist auf folgende Beziehungen hin: Prof. Dr. Korting kooperiert mit Unternehmen der kosmetischen Industrie bei der Herstellung von Kosmetika. 